\title{
Selection of Some Promising Guava Trees Grown in Kafr El-Sheikh Governorate
}

\author{
B. M. Khalil", E. A. H. Ahmed", Salwa El-Habashy ${ }^{*}$, Nahla \\ A. Awd" and M. A. El-Hamady ${ }^{* *}$ \\ *Fruit Breeding Department, Horticulture Research Institute, \\ Agricultural Research Centre, Cairo and ${ }^{* *}$ Faculty of Agriculture \\ Kafr El-Sheikh University, Kafr El-Sheikh, Egypt.
}
CUAVA (Psidium guajava L.), is one of the most common fruits in T. tropical and subtropical regions. It could be used for both fresh consumption and processing. Moreover, guava has been cultivated in Egypt for a long time, minimal research on value added has been conducted. Therefore, there is a real demand for selections of promising guava strains to increase production, processing and marketing.

\begin{abstract}
Nineteen superior guava strains were selected and labeled in their orchards (A, B, C and D), in Kafr El-Sheikh Governorate, according to fruit quality traits in two successive seasons (2013 and 2014). Intensive survey has been done on yielding, fruit physical and chemical properties. Some selected strains exhibited a highest fruit weight during the two seasons, such as strains A3 and C7, which recorded (356.70 and $405.00 \mathrm{~g}$, respectively) in the first season and (392.30 and $431.80 \mathrm{~g}$, respectively) in the second season. Strain D16 recorded a good firmness in the two seasons (10.86 to $10.89 \mathrm{Lb} / \mathrm{I}^{2}$, respectively). Strains A1 and D18 showed the lowest number of seeds per fruit in the first season (100 and 113 seeds, respectively) and (105 and 127 seeds, respectively) in the second season. Fruit chemical characteristics of the nineteen selected guava strains, revealed that the selected strain $\mathrm{C} 8$ gave highest values of soluble solid contents in the two seasons $\left(14.00\right.$ and $14.17^{\circ}$ Brix, respectively). Two selected strains $\mathrm{C} 11$ and $\mathrm{C} 12$ gave the highest value of SSC/acidity ratio (75.81 and 76.39) in season 2013 and (69.81 and 71.04) in season 2014. A highest value of vitamin $\mathrm{C}$ was recorded for selected strains (B5, C7, C8, C9, C13 and C16).
\end{abstract}

Keywords: Guava, Production, Selection, Strains.

Guava (Psidium guajava L.), belongs to Myrtaceae family, with approximately 130 genera and 3000 species of trees and shrubs distributed mainly in the tropics and subtropics. Guava is native from the Americas, but was introduced to other regions of the world, where it is cultivated nowadays. It constitute one of the tropical and subtropical fruits of great nutritional value, due to their vitamin and mineral contributions. It gained recognition as valuable sources of ascorbic acid (Risterucci et al., 2005).

Guava trees is drought-tolerant, grow rapidly and fruit in two to four years from seed. They live 30 to 40 years but productivity declines after the $15^{\text {th }}$ year. Drastic pruning may rejuvenate orchards. The fruits of guava are very variable in 
size and flavor, ranging from sweet to tart, and all have a characteristic penetrating musky odor, which is more marked in some forms than others are. Ripe juicy sweet types are eaten fresh. It contains 2-5 times the vitamin C content of fresh orange juice (Purseglove, 1984).

Due to the great variability of guavas raised from seeds, so vegetative propagation of superior clones is highly desirable. In Egypt, there is a great variability in type of fruits, yield and fruit ripening, since most guava orchards are produced by seeds. Growers asks for high yielding clonal varieties of guava, with good eating qualities. El-Wakeel et al. (1971) studied the seedling population of guava trees, and stated that some progenies varied in time of blooming, time of harvest and fruit characters, and initiated selection of guava in Alexandria and Beheira governorates.

'Bassateen El Sabahia' guava cultivar has long been the standard commercial guava. Moreover, 'Bassateen Edfina' guava cultivar was the result of selections from three hundred seedlings. It was tested and introduced into cultivation in 1975. It was promising cultivars in yield of medium size pear-shaped fruits, with thick white flesh, few seeds, good flavor and higher ascorbic acid content, sometimes pink-blushed (Rokba et al., 1976).

Fruit with lots of seeds makes guava suitable for controlled hybridization, also the resulting progenies of open pollination can be appropriate for development programs of cultivars (Nakasone and Paull 1998).

El-Hamady et al. (2002) evaluated, characterized and established the relationship among some superior selected seedling guava strains by using RAPD analysis, and found enough genetic variability to warrant the possibility of guava improvement.

Many breeding programs in the world have released improved guava cultivars, but the most common way of getting new varieties is through grower's actions, such as identification of outstanding plants in their orchards and their propagation. This is possible because of the great diversity in open pollinated plants used to form orchards. There are probably more than 400 guava cultivars around the world, but only a few dozen are responsible for the majority of plantings, such as [Allahabad Safeda, Karela, Safeda] in India, [Paluma, Rica, Pedro Sato] in Brazil (Pommer and Murakami, 2009).

The main objective of the present study is to select some superior seedling guava strains from private orchards in Kafr El-Sheikh Governorate on the base of yielding and fruit characteristics to be a good start in breeding program. 


\section{Materials and Methods}

A breeding program was initiated in 2010 by Fruit Breeding Dept., Hort. Res. Inst. Agric. Res. Center in Egypt, to select good strains of guava (Psidium guajava L.).

This study was carried out during two growing seasons 2013 and 2014. Nineteen superior guava trees were selected from seedling population (propagated by seeds) grown in four private orchards (A, B, C and D) in the north of Kafr El-Sheikh Governorate, according to visual evaluation, quality traits and yield. The geographical coordinates of these locations are $31^{\circ} 33^{\prime} 34^{\prime \prime}$ North, $31^{\circ} 05^{`} 30^{\prime \prime}$ East.

Samples of fifteen fruits per tree were collected randomly then transported to the laboratory to determine physical and chemical characteristics as follow:

\section{Physical fruit characteristics}

Fruit samples were weighted and the average fruit weight for each replicate was calculated. Diameter and length of fruits were measured using a hand caliper. Fruit firmness was measured using the fruit pressure tester model (FT 327) with $8 \mathrm{~mm}$ plunger [EFFEGI, 48011 Alfonsine, Italy]. Flesh thickness were determined. Weight of 100 seeds, total weight of seeds and total number of seeds per fruit were recorded.

\section{Chemical fruit characteristics}

According to the official methods of analysis (A.O.A.C. 1990), Soluble solids content (SSC ${ }^{\circ}$ Brix) was determined using ABBE Refractometer at the room temperature, Total acidity percentage of the juice was measured, and the ascorbic acid (vitamin C) was determined by the dye method of 2,6dichlorophenol indophenol.

\section{Statistical analysis}

Experiment was designed in completely randomized design. From each selected strains, fifteen fruits was picked randomly and divided into three replicates. Data were subjected to analysis of variance. Duncan's multiple range test at $5 \%$ level of significance $(p=0.05)$ was used for means comparisons according to Gomez and Gomez (1984).

\section{Results and Discussion}

Differences of physical fruit characteristics in the two successive seasons (2013 and 2014) were illustrated in Tables 1a and 1b. Statistical analysis revealed that significant differences occurred in weight of fruits. The highest fruit weight was obtained by strain $\mathrm{C} 7$ that recorded 405.00 and $431.80 \mathrm{~g}$ during both seasons, respectively, while, the lowest weight was 82.00 and $90.20 \mathrm{~g}$ recorded by strain A1 during both seasons, respectively. The other fruit weights were ranged between (167.00 and $356.70 \mathrm{~g}$ ) for strains $\mathrm{C} 11$ and $\mathrm{A} 3$, respectively in the first season, and from (187.30 to $392.30 \mathrm{~g}$ ) for strains $\mathrm{C} 12$ and $\mathrm{A} 3$, respectively in the second season. Regarding the fruit size, strain $\mathrm{C} 7$ raised the highest values 413.30 and $443.30 \mathrm{~cm}^{3}$, while, strain A1 recorded the lowest values 81.00 and $89.00 \mathrm{~cm}^{3}$, during the two seasons, respectively. Significant variation was detected in fruit length for all selected strains. Strain C7 score the 
highest values $(85.53$ and $87.87 \mathrm{~mm})$, while strain A1 had the lowest values (50.93 and $53.11 \mathrm{~mm})$, during the two seasons, respectively. Other values ranged from 56.16 to $82.90 \mathrm{~mm}$ in the first season and from 58.72 to $84.90 \mathrm{~mm}$ in the second season for D19 and A3, respectively.

Concerning the fruit diameter, Table 1a showed that the same strain C7 gave the highest values $(93.90$ and $96.23 \mathrm{~mm})$, while, strain A1 had the lowest values (49.13 and $51.47 \mathrm{~mm}$ ) during the two seasons, respectively. The other values were ranged from 67.13 to $83.73 \mathrm{~mm}$ for strains D19 and D15, respectively in season 2013, while in season 2014, values were ranged from 69.47 to $88.87 \mathrm{~mm}$ for strains D19 and A3, respectively. In respect to fruit firmness, significant differences were recorded between all selected strains, the highest value was $\left(10.89 \mathrm{lb} / \mathrm{I}^{2}\right)$ for selected strain D16, while, strains C12, C11, C13 and C10 showed the lowest values, $\left(2.60,2.67,2.73\right.$ and $2.77 \mathrm{lb} / \mathrm{I}^{2}$, respectively). The other selected strains recorded values ranged from 9.07 to $3.54 \mathrm{lb} / \mathrm{I}^{2}$ in the first season. The same trend was achieved in the second season with strain D16 that scored the highest value $\left(10.86 \mathrm{lb} / \mathrm{I}^{2}\right)$ while strains $\mathrm{C} 12, \mathrm{C} 13$ and $\mathrm{C} 11$ showed the lowest values, $\left(2.80,2.84\right.$ and $2.87 \mathrm{lb} / \mathrm{I}^{2}$, respectively). Low firmness is against marketing of guava fruits due to the poor harvest handling that lead to uncommercial production. Therefore, developing of appropriate technologies for production, processing of guava fruits and utilization of its products are necessary (Gatambia et al., 2009).

Table $1 \mathrm{~b}$ showed a considerable variation in values of flesh thickness for all selected strains. The highest values were observed among strain C13 $(2.00 \mathrm{~cm})$ in season 2013 and among strain C14 $(2.10 \mathrm{~cm})$ in season 2014, while, the lowest values $(1.27$ and $1.32 \mathrm{~cm})$ were achieved by strain A1 during the two seasons, respectively.

Table $1 \mathrm{~b}$ showed the weight of 100 seeds for all selected strains. Significant differences were recorded among these values. The highest values were 2.56 and $2.67 \mathrm{~g}$ for strain B5, while, the lowest values were scored by strain C11 (0.96 and $1.16 \mathrm{~g}$ ) during the two seasons, respectively. These results were in agreement with Patel et al., (2011), who found that the minimum weight of 100 seeds was $0.96 \mathrm{~g}$.

Statistical analysis revealed that significant differences were occurred in the total weight of seeds per fruit as shown in Table 1b. Selected strain C7 recorded the highest values $(8.05$ and $8.31 \mathrm{~g})$, while, the lowest values were (1.90 and $2.10 \mathrm{~g}$ ) for the selected strain D18, during the two seasons, respectively. Other strains scored values intermediate. Concerning the number of seeds per fruit, the highest number was (405 and 420) for selected strain A3 and (385 and 403) for selected strain $\mathrm{C} 7$ with insignificant differences between the two selected strains during the two seasons, while, the lowest number was (100 and 105) for strain A1 during the two seasons, respectively. Moreover, the other strains recorded intermediate values. It is a fact that fruit quality of guava was better when its content of seeds decreased according to Nakasone and Paull (1998), who stated that the selection criteria of guava fruits are, large fruits (200-340 g) with few seeds, thick pulp, high content of total soluble solids and low acidity percentage.

Egypt. J. Hort. Vol. 42, No. 1 (2015) 


\begin{tabular}{|c|c|c|c|c|c|c|c|c|c|c|c|c|c|c|c|c|c|c|c|c|}
\hline \multirow{2}{*}{ 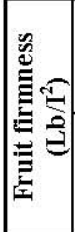 } & जิ & $\mid \begin{array}{l}\frac{1}{0} \\
0 \\
0 \\
\forall \\
\forall\end{array}$ & $\begin{array}{l}0 \\
\dot{0} \\
2 \\
n \\
n\end{array}$ & 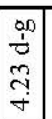 & \begin{tabular}{|c|c}
0 \\
0 \\
0 \\
+ \\
+
\end{tabular} & 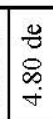 & $\left|\begin{array}{c}0 \\
0 \\
7 \\
\end{array}\right|$ & $\begin{array}{l}\overrightarrow{0} \\
b \\
n\end{array}$ & 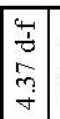 & 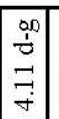 & $\begin{array}{c}\stackrel{20}{\leftrightarrows} \\
\vec{m}\end{array}$ & \begin{tabular}{c|c}
$\infty$ & \\
$\infty$ & \\
$\infty$ & \\
&
\end{tabular} & 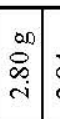 & 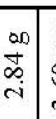 & 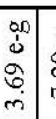 & ڤె் & 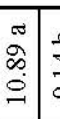 & & & $\frac{8}{8}$ \\
\hline & 을 & $\begin{array}{l}\stackrel{8}{0} \\
\stackrel{0}{p} \\
\dot{\sim}\end{array}$ & 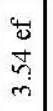 & $\begin{array}{l}\frac{w}{y} \\
\dot{0} \\
\dot{\sigma}\end{array}$ & 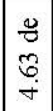 & $\begin{array}{l}\frac{8}{8} \\
8 \\
\dot{8}\end{array}$ & 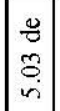 & $\begin{array}{c}\vec{z} \\
\dot{q} \\
\dot{v}\end{array}$ & 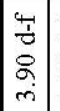 & \begin{tabular}{|c|} 
\\
\\
0 \\
0 \\
$\dot{f}$ \\
\end{tabular} & $\underset{i}{\stackrel{4}{a}}$ & 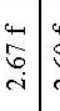 & 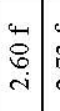 & $\begin{array}{l}\stackrel{\leftrightarrow}{c} \\
\stackrel{i}{i}\end{array}$ & 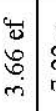 & ב্خ & 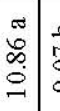 & & & $\begin{array}{l}8 \\
8 \\
8 \\
\\
0 \\
0\end{array}$ \\
\hline \multirow{2}{*}{ 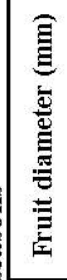 } & $\overrightarrow{\tilde{N}}$ & $\left|\begin{array}{c}+ \\
f \\
\vec{r} \\
\dot{n}\end{array}\right|$ & $\begin{array}{c}0 \\
\dot{0} \\
2 \\
2 \\
2\end{array}$ & $\begin{array}{c}\approx \\
\tilde{\sigma} \\
\infty \\
\infty \\
\infty \\
\infty\end{array}$ & 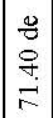 & 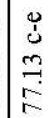 & $\mid \begin{array}{c}T \\
\dot{b} \\
0 \\
0 \\
i \\
\infty\end{array}$ & 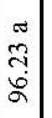 & $\mid \begin{array}{c}2 \\
\dot{1} \\
\tilde{2} \\
\tilde{n} \\
2\end{array}$ & 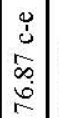 & $\begin{array}{l}\mathscr{D} \\
0 \\
0 \\
\dot{\infty}\end{array}$ & $\begin{array}{c}\dot{u} \\
\dot{u} \\
\dot{g} \\
r\end{array}$ & $\begin{array}{l}0 \\
\dot{0} \\
\dot{0} \\
\dot{\varepsilon}\end{array}$ & 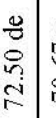 & & 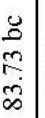 & $\begin{array}{c}8 \\
8 \\
8 \\
\dot{8} \\
\dot{c} \\
\infty\end{array}$ & & & 寄 \\
\hline & 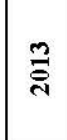 & 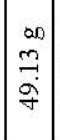 & $\mid \begin{array}{l}4 \\
0 \\
0 \\
f \\
0 \\
0\end{array}$ & $\left|\begin{array}{l}0 \\
0 \\
0 \\
0 \\
\infty \\
\infty\end{array}\right|$ & 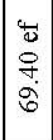 & 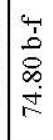 & 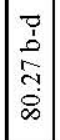 & $\begin{array}{l}\approx \\
\check{\alpha} \\
\tilde{\sigma}\end{array}$ & $\left|\begin{array}{l}\vec{r} \\
\tilde{c} \\
\hat{n} \\
\tilde{F}\end{array}\right|$ & 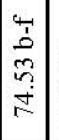 & $\begin{array}{l}\dot{0} \\
\dot{0} \\
\hat{0} \\
\infty \\
\infty\end{array}$ & 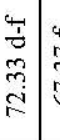 & $\begin{array}{l}4 \\
\frac{4}{3} \\
\frac{6}{6}\end{array}$ & 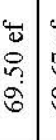 & 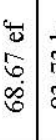 & $\begin{array}{c}\stackrel{0}{m} \\
\stackrel{\infty}{\infty}\end{array}$ & 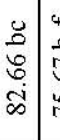 & & & \\
\hline \multirow{2}{*}{ 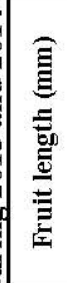 } & ت্ّ & $\begin{array}{l}+ \\
= \\
\dot{m}\end{array} \mid$ & $\mid$\begin{tabular}{c|c}
0 \\
0 \\
0 \\
0 \\
$w$ \\
2 \\
2
\end{tabular} & 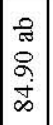 & $\mid \begin{array}{l}\vec{T} \\
\dot{b} \\
\stackrel{2}{2} \\
\dot{d}\end{array}$ & 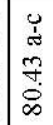 & 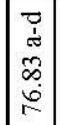 & $\begin{array}{l}\infty \\
\infty \\
\infty \\
\infty \\
\infty\end{array}$ & 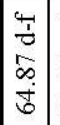 & 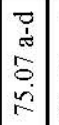 & 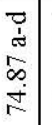 & 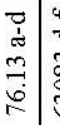 & 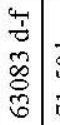 & 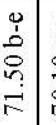 & $\begin{array}{l}\dot{d} \\
\dot{\delta} \\
\stackrel{0}{0} \\
\dot{0}\end{array}$ & 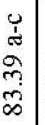 & 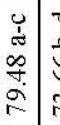 & & & 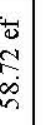 \\
\hline & 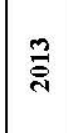 & 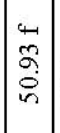 & $\mid \begin{array}{l}0 \\
0 \\
0 \\
\tilde{\alpha} \\
0 \\
0\end{array}$ & $\begin{array}{l}\text { न } \\
\stackrel{\Omega}{ } \\
\stackrel{\infty}{\infty} \\
\infty\end{array}$ & $\begin{array}{l}\vec{T} \\
\dot{b} \\
\stackrel{0}{0} \\
\stackrel{i}{r}\end{array}$ & 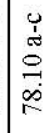 & 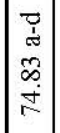 & $\begin{array}{l}\approx \\
\tilde{n} \\
\infty \\
\infty\end{array}$ & 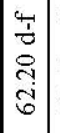 & 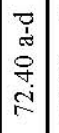 & 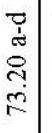 & 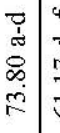 & $\begin{array}{l}4 \\
1 \\
5 \\
-6 \\
6\end{array}$ & 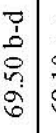 & $\begin{array}{c}\dot{i} \\
\dot{\delta} \\
\vdots \\
\dot{0} \\
\dot{0}\end{array}$ & 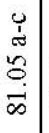 & 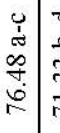 & & & 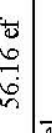 \\
\hline 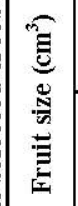 & 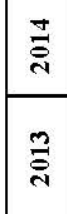 & $\mid \begin{array}{c}\infty \\
8 \\
0 \\
\infty \\
\infty\end{array}$ & 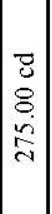 & $\begin{array}{l}0 \\
0 \\
0 \\
6 \\
e n \\
e n\end{array}$ & 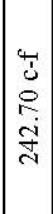 & $\mid \begin{array}{l}0 \\
\dot{\delta} \\
\delta \\
\delta \\
\hat{z}\end{array}$ & 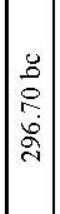 & 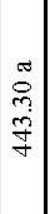 & 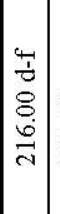 & 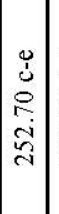 & 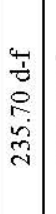 & 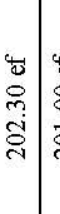 & 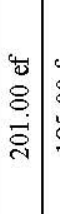 & 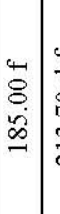 & $\begin{array}{l}\frac{W}{0} \\
\stackrel{0}{0} \\
\vec{n} \\
\vec{d}\end{array}$ & 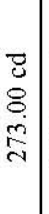 & 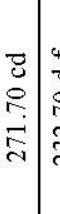 & 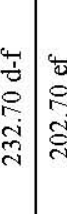 & & 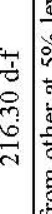 \\
\hline & تั & $\left|\begin{array}{c}\infty \\
\delta \\
\dot{\infty} \\
\mid\end{array}\right|$ & \begin{tabular}{|c|}
$\overrightarrow{0}$ \\
8 \\
8 \\
$\dot{0}$ \\
\end{tabular} & \begin{tabular}{|l|}
0 \\
0 \\
0 \\
0 \\
0 \\
0
\end{tabular} & 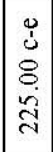 & $\begin{array}{l}\dot{y} \\
\dot{\delta} \\
8 \\
\dot{0} \\
\tilde{N}\end{array}$ & \begin{tabular}{|c|}
0 \\
8 \\
8 \\
$\vdots$ \\
\\
\end{tabular} & 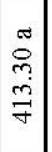 & 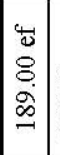 & $\mid \begin{array}{l}0 \\
\dot{0} \\
0 \\
i \\
\tilde{N} \\
\mid\end{array}$ & $\begin{array}{l}\vec{t} \\
0 \\
8 \\
\dot{\vec{t}} \\
\vec{\lambda}\end{array}$ & 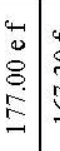 & 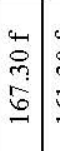 & 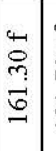 & 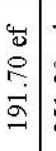 & 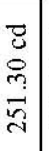 & 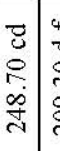 & 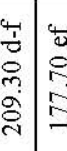 & & 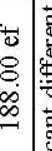 \\
\hline & $\stackrel{m}{\stackrel{\sim}{*}}$ & 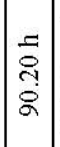 & 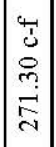 & 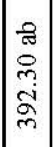 & 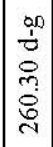 & $\begin{array}{l}\frac{0}{1} \\
\frac{1}{2} \\
\stackrel{\rho}{\sigma} \\
\frac{d}{d}\end{array}$ & $\mid \begin{array}{l}0 \\
\dot{\jmath} \\
0 \\
\dot{8} \\
\dot{0} \\
\mid\end{array}$ & 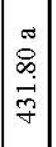 & 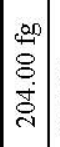 & 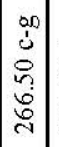 & 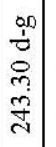 & 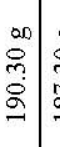 & $\begin{array}{c}\infty \\
\infty \\
0 \\
0 \\
\vdots \\
-\infty \\
-1\end{array}$ & 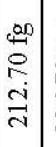 & 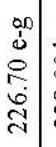 & 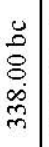 & 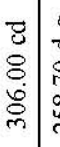 & 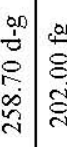 & & 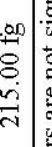 \\
\hline & & $\mid \begin{array}{c}\vec{\Xi} \\
\stackrel{8}{ } \\
\infty\end{array}$ & 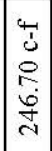 & $\mid \begin{array}{l}\tilde{\epsilon} \\
0 \\
0 \\
0 \\
m \\
m\end{array}$ & 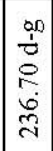 & 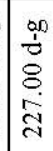 & 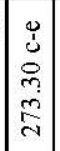 & 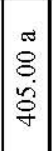 & $\left|\begin{array}{c}\infty \\
8 \\
8 \\
\infty \\
\infty \\
-1\end{array}\right|$ & 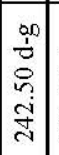 & 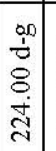 & 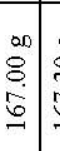 & 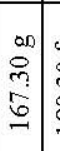 & 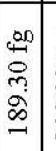 & 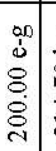 & 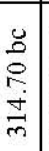 & 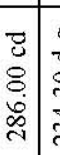 & 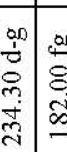 & & $\begin{array}{c}0 \\
8 \\
8 \\
\infty \\
\infty \\
-\infty \\
-1\end{array}$ \\
\hline & & -1 & $\sim$ & $m$ & F & in & 6 & $r$ & $\infty$ & $a$ & $\stackrel{-}{-}$ & $\Rightarrow=$ & $\approx$ & 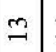 & \pm & -1 & $\div$ & $=10$ & & $\approx$ \\
\hline & & & $\varangle$ & & & m & & & & & & & & & & & & $\theta$ & & \\
\hline
\end{tabular}


TABLE 1 (b). Fruit physical characteristics of different selected trees during 2013 and 2014 seasons

\begin{tabular}{|c|c|c|c|c|c|c|c|c|c|}
\hline \multirow{2}{*}{ Farm } & \multirow{2}{*}{$\begin{array}{l}\text { No. of } \\
\text { strain }\end{array}$} & \multicolumn{2}{|c|}{$\begin{array}{l}\text { Flesh thickness } \\
\text { (cm) }\end{array}$} & \multicolumn{2}{|c|}{$\begin{array}{l}\text { Weight of } 100 \\
\text { seeds (g) }\end{array}$} & \multicolumn{2}{|c|}{$\begin{array}{l}\text { Total weight of } \\
\text { seeds }(\mathrm{g})\end{array}$} & \multicolumn{2}{|c|}{$\begin{array}{l}\text { Total number } \\
\text { of seeds per } \\
\text { fruit }\end{array}$} \\
\hline & & 2013 & 2014 & 2013 & 2014 & 2013 & 2014 & 2013 & 2014 \\
\hline \multirow{3}{*}{ A } & 1 & $1.27 \mathrm{~g}$ & $1.32 \mathrm{~g}$ & $2.43 \mathrm{ab}$ & $2.55 \mathrm{a}$ & $2.43 \mathrm{~g}$ & $2.66 \mathrm{f}$ & $100 \mathrm{~h}$ & $105 \mathrm{j}$ \\
\hline & 2 & $1.63 \mathrm{c}-\mathrm{e}$ & $1.70 \mathrm{~b}-\mathrm{e}$ & $1.72 \mathrm{c}-\mathrm{e}$ & $1.81 \mathrm{~b}-\mathrm{e}$ & $6.13 b$ & $6.45 \mathrm{~b}$ & $360 \mathrm{ab}$ & $376 a b$ \\
\hline & 3 & $1.50 \mathrm{ef}$ & $1.43 \mathrm{fg}$ & $1.52 \mathrm{c}-\mathrm{f}$ & $1.59 \mathrm{~b}-\mathrm{e}$ & $6.20 \mathrm{~b}$ & $6.40 \mathrm{~b}$ & $405 \mathrm{a}$ & $420 \mathrm{a}$ \\
\hline \multirow{3}{*}{ B } & 4 & $1.56 \mathrm{~d}-\mathrm{f}$ & $1.65 \mathrm{c}-\mathrm{e}$ & $2.15 \mathrm{a}-\mathrm{c}$ & $2.24 \mathrm{ab}$ & $4.82 \mathrm{c}$ & $4.99 \mathrm{c}$ & $226 \mathrm{f}$ & $246 \mathrm{~h}$ \\
\hline & 5 & $1.63 \mathrm{c}-\mathrm{e}$ & $1.71 \mathrm{~b}-\mathrm{e}$ & $2.56 \mathrm{a}$ & $2.67 \mathrm{a}$ & $5.76 \mathrm{~b}$ & $5.99 \mathrm{~b}$ & $255 \mathrm{ef}$ & $268 \mathrm{f}-\mathrm{h}$ \\
\hline & 6 & $1.63 \mathrm{c}-\mathrm{e}$ & $1.60 \mathrm{~b}-\mathrm{e}$ & $1.92 \mathrm{a}-\mathrm{d}$ & $2.08 \mathrm{a}-\mathrm{c}$ & $5.70 \mathrm{~b}$ & $5.89 \mathrm{~b}$ & $296 \mathrm{c}-\mathrm{e}$ & $310 \mathrm{c}-\mathrm{g}$ \\
\hline \multirow{8}{*}{$\mathrm{C}$} & 7 & 1.50 ef & $1.66 \mathrm{c}-\mathrm{e}$ & $2.09 \mathrm{a}-\mathrm{c}$ & $2.28 \mathrm{ab}$ & $8.05 \mathrm{a}$ & $8.31 \mathrm{a}$ & $385 \mathrm{a}$ & $403 \mathrm{a}$ \\
\hline & 8 & $1.56 \mathrm{~d}-\mathrm{f}$ & $1.66 \mathrm{c}-\mathrm{e}$ & $1.53 \mathrm{c}-\mathrm{f}$ & $1.65 \mathrm{~b}-\mathrm{e}$ & $3.80 \mathrm{~d}-\mathrm{f}$ & $4.03 \mathrm{de}$ & 254 ef & $273 \mathrm{f}-\mathrm{h}$ \\
\hline & 9 & $1.50 \mathrm{ef}$ & $1.60 \mathrm{~d}-\mathrm{f}$ & $1.81 \mathrm{~b}-\mathrm{d}$ & $2.01 \mathrm{a}-\mathrm{c}$ & $4.82 \mathrm{c}$ & $5.05 \mathrm{c}$ & $270 \mathrm{~d}-\mathrm{f}$ & 286 d-h \\
\hline & 10 & $1.60 \mathrm{c}-\mathrm{f}$ & $1.68 \mathrm{c}-\mathrm{e}$ & $1.25 \mathrm{~d}-\mathrm{f}$ & $1.44 \mathrm{c}-\mathrm{e}$ & $3.60 \mathrm{~d}-\mathrm{f}$ & $3.86 \mathrm{de}$ & 303 b-e & $319 b-f$ \\
\hline & 11 & $1.73 \mathrm{bc}$ & $1.82 \mathrm{bc}$ & $0.96 \mathrm{f}$ & $1.16 \mathrm{e}$ & $3.10 \mathrm{f}$ & $3.40 \mathrm{e}$ & $328 \mathrm{bc}$ & $341 \mathrm{~b}-\mathrm{d}$ \\
\hline & 12 & $1.80 \mathrm{~b}$ & $1.88 \mathrm{~b}$ & $1.50 \mathrm{c}-\mathrm{f}$ & $1.63 \mathrm{~b}-\mathrm{e}$ & $3.66 \mathrm{~d}-\mathrm{f}$ & $3.89 \mathrm{de}$ & 246 ef & $364 \mathrm{a}-\mathrm{c}$ \\
\hline & 13 & $2.00 \mathrm{a}$ & $1.60 \mathrm{~d}-\mathrm{f}$ & $1.07 \mathrm{ef}$ & $1.26 \mathrm{de}$ & $3.20 \mathrm{ef}$ & $3.43 \mathrm{de}$ & $321 \mathrm{~b}-\mathrm{d}$ & 334 b-e \\
\hline & 14 & $1.50 \mathrm{ef}$ & $2.10 \mathrm{a}$ & $1.57 \mathrm{c}-\mathrm{f}$ & $1.65 \mathrm{~b}-\mathrm{e}$ & $3.40 \mathrm{~d}-\mathrm{f}$ & $3.63 \mathrm{de}$ & $231 \mathrm{f}$ & $245 \mathrm{~h}$ \\
\hline \multirow{5}{*}{ D } & 15 & 1.49 ef & $1.57 \mathrm{~d}-\mathrm{f}$ & $1.73 \mathrm{c}-\mathrm{e}$ & $1.89 \mathrm{~b}-\mathrm{d}$ & $3.90 \mathrm{de}$ & $4.13 \mathrm{de}$ & $220 \mathrm{f}$ & $235 \mathrm{hi}$ \\
\hline & 16 & $1.47 \mathrm{f}$ & $1.53 \mathrm{ef}$ & $1.57 \mathrm{c}-\mathrm{f}$ & $1.66 \mathrm{~b}-\mathrm{e}$ & $4.00 \mathrm{~d}$ & $4.20 \mathrm{~d}$ & $270 \mathrm{~d}-\mathrm{f}$ & $281 \mathrm{e}-\mathrm{h}$ \\
\hline & 17 & $1.32 \mathrm{~g}$ & $1.45 \mathrm{fg}$ & $1.57 \mathrm{c}-\mathrm{f}$ & $1.65 \mathrm{~b}-\mathrm{e}$ & $3.50 \mathrm{~d}-\mathrm{f}$ & $3.73 \mathrm{de}$ & $236 \mathrm{f}$ & $252 \mathrm{gh}$ \\
\hline & 18 & $1.66 \mathrm{~b}-\mathrm{d}$ & $1.75 \mathrm{~b}-\mathrm{d}$ & $1.69 \mathrm{c}-\mathrm{e}$ & $1.85 \mathrm{~b}-\mathrm{e}$ & $1.90 \mathrm{~g}$ & $2.10 \mathrm{f}$ & $113 \mathrm{gh}$ & $127 \mathrm{j}$ \\
\hline & 19 & 1.50 ef & $1.60 \mathrm{~d}-\mathrm{f}$ & $2.07 \mathrm{a}-\mathrm{c}$ & $2.26 \mathrm{ab}$ & 3.22 ef & $3.42 \mathrm{e}$ & $164 \mathrm{~g}$ & $184 \mathrm{i}$ \\
\hline
\end{tabular}

Means followed by the same letters are not significant different from other at 5\% level.

Table 2 showed the fruit chemical of nineteen selected strains of guava in 2013 and 2014 seasons. Significant differences existed with SSC values and total acidity percentage, during the two seasons. The highest values (14.00 and 14.17 ${ }^{\circ}$ Brix) of SSC were for selected strain C8, while, the lowest values were scored by strain D16 (6.67 and $6.83{ }^{\circ}$ Brix) during the two seasons, respectively. Moreover, SSC values for the other selected strains recorded intermediate values. Concerning the acidity percentage, significant differences were recorded between the different selected strains of guava, the highest value $(0.53 \%)$ was for strain

Egypt. J. Hort. Vol. 42, No. 1 (2015) 
A2 in the first season, while, it was $(0.44 \%)$ for selected strain A3 in the second season. The lowest values were achieved by selected strains C7, C11, C12, D15, D16, D17, D18 and D19 with insignificant differences, which ranged from 0.16 to $0.21 \%$ in the first season and from 0.18 to $0.21 \%$ in the second season.

Regarding SSC/acidity ratio, significant differences were recorded among all selected strains of guava. Selected strains $\mathrm{C} 11$ and $\mathrm{C} 12$ raised the highest ratio during the two seasons with insignificant differences, C11 strain scored ratio of (76.39 and 71.04) while, strain C12 sored ratio of (75.81 and 69.81) in 2013 and 2014 seasons, respectively. In addition, the lowest ratio in the first season was (22.15 and 22.50) for selected strains A2 and B6, respectively, while, in the second season, strain B6 was recorded the lowest significant ratio (21.64).

Purseglove (1984) stated that the total soluble solids in the mesocarp of some improved cultivars in Hawaii is about 10 percent and the white flesh has a sweet acid flavor and may be eaten raw or made into jam and jelly in Brazil.

The contents of vitamin $\mathrm{C}$ in $100 \mathrm{ml}$ of guava juice for all selected strains were presented in Table 2. In the first season, selected strains C7, C8 and C9 recorded the highest values with insignificant differences, (121.00, 113.00 and $114.00 \mathrm{mg}$, respectively). While, selected strain C7 gave the highest value $(124.60 \mathrm{mg})$ in the second season, on the other hand, the selected strain D18 recorded the lowest values (47.33 and $48.33 \mathrm{mg})$ in the two seasons, respectively. Other selected strains gave intermediate values during the two seasons.

Purseglove (1984) stated that guava are particularly rich in vitamin C, which its ascorbic acid contents varied from 23 to $486 \mathrm{mg}$ per $100 \mathrm{~g}$ of fresh fruit in Florida, and ranged from 146 to $492 \mathrm{mg}$ with an average of $261 \mathrm{mg}$ for eight selected cultivars propagated vegetatively in Hawaii.

\section{Conclusion}

In Egypt, guava crop is neglected by research and development strategies, in spite of its high nutrition value and it can be used as an important resource for our local community due to their great potential for income generation. Therefore, there is a real demand to select promising guava strains in yielding and good fruit characteristics for different consumptions, to increase its production, processing and marketing. Moreover, more breeding programs are needed to introduce and evaluate many superior cultivars for different uses. In present data, our survey in private orchards of guava in the north of Kafr ElSheikh Governorate, there are nineteen selected strains with high yield and good quality of fruits. The selected strains A3, C7, C9 and D15 had the highest fruit weight and size, then its yield. Other selected strains C11 and C12 had the highest SSC/acidity ratio. Some of these selected strains had highest content of vitamin $\mathrm{C}$ in the fruit such as B5, C7, C8, C9, C13 and D16 strains. The selected strain A1 has a small fruit with few seeds and moderate vitamin $\mathrm{C}$ content. Figures 1 and 2 showed some selected strains from four private orchards A, B, C and D. 


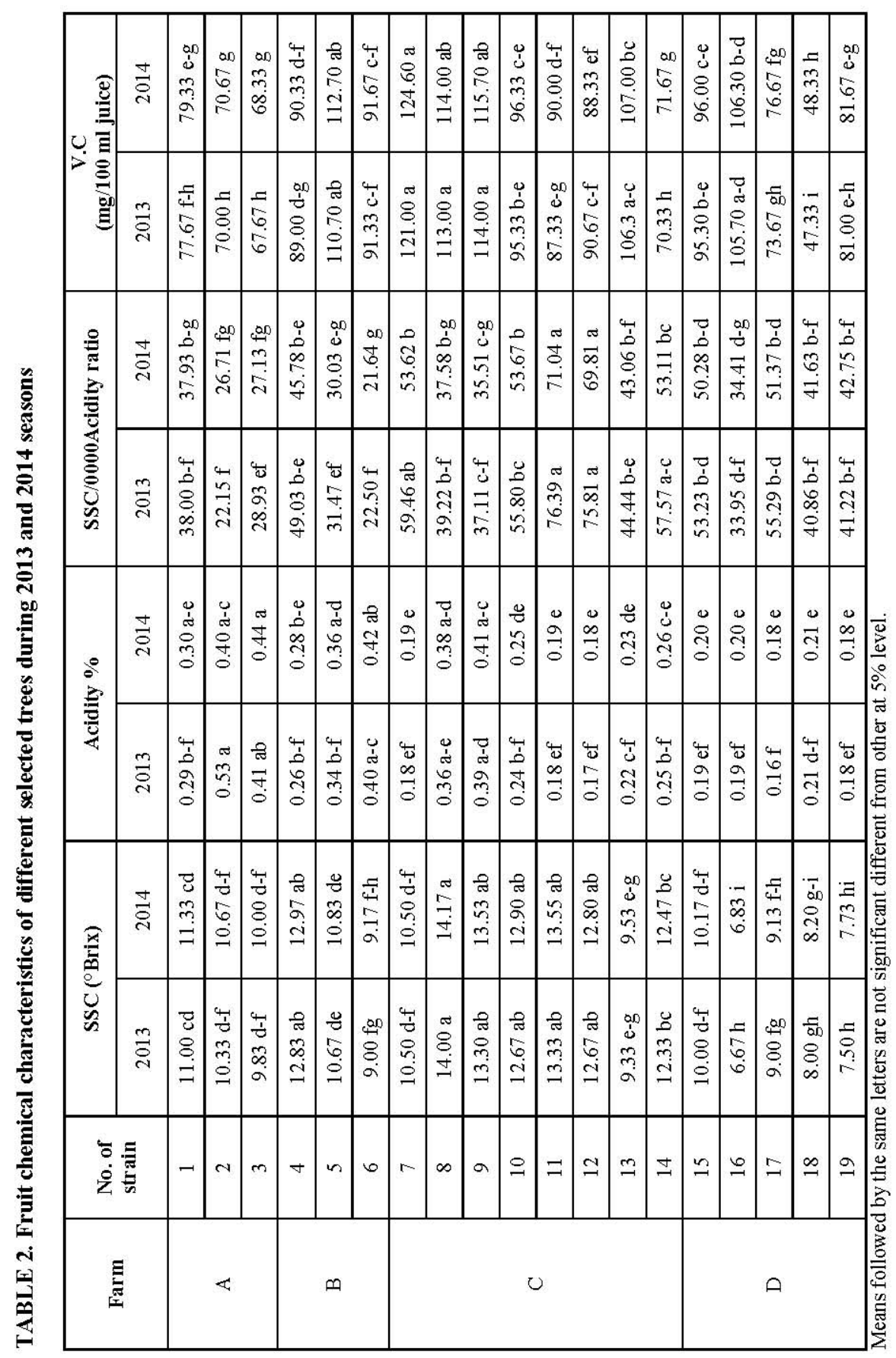

Egypt. J. Hort. Vol. 42, No. 1 (2015) 
Finally, the nineteen selected strains of guava were propagated vegetatively by budding and grafting on seedling rootstocks in their orchards to study their vegetative growth, morphological characters, total yield per tree for each strain and fruit characters for the demands of different uses (fresh use and industrial process). There is a need to develop these selected guava strains and to study their genetic variability at the molecular level. In addition, more research may be carried out through modern techniques.

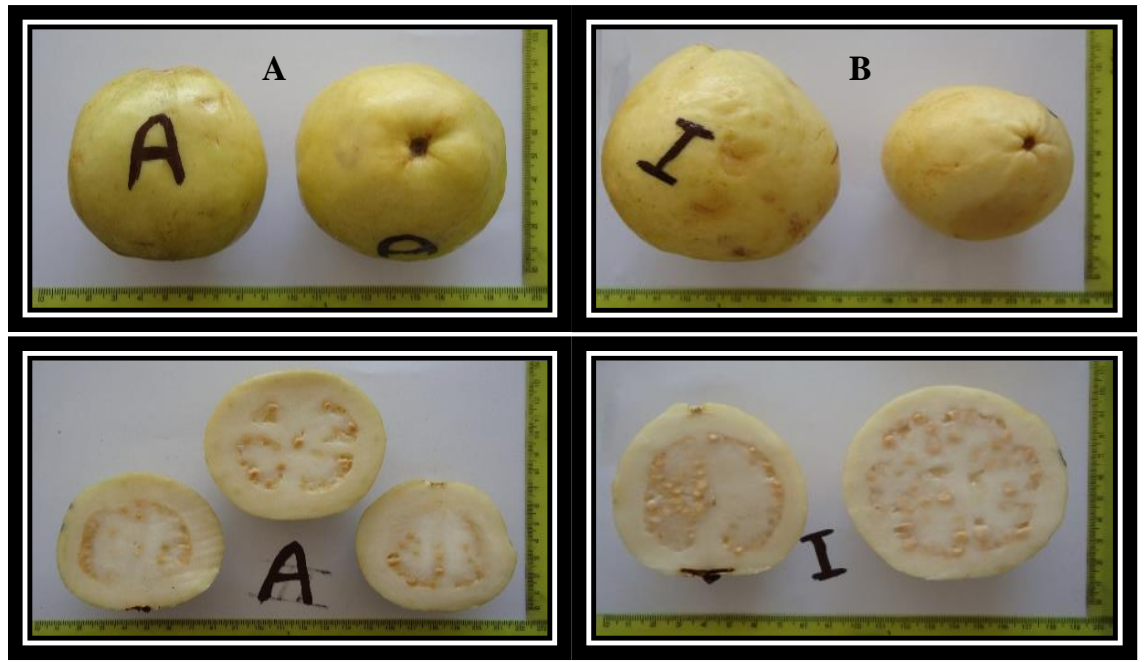

Fig. 1. Guava fruit of some selected strains from orchards A and B
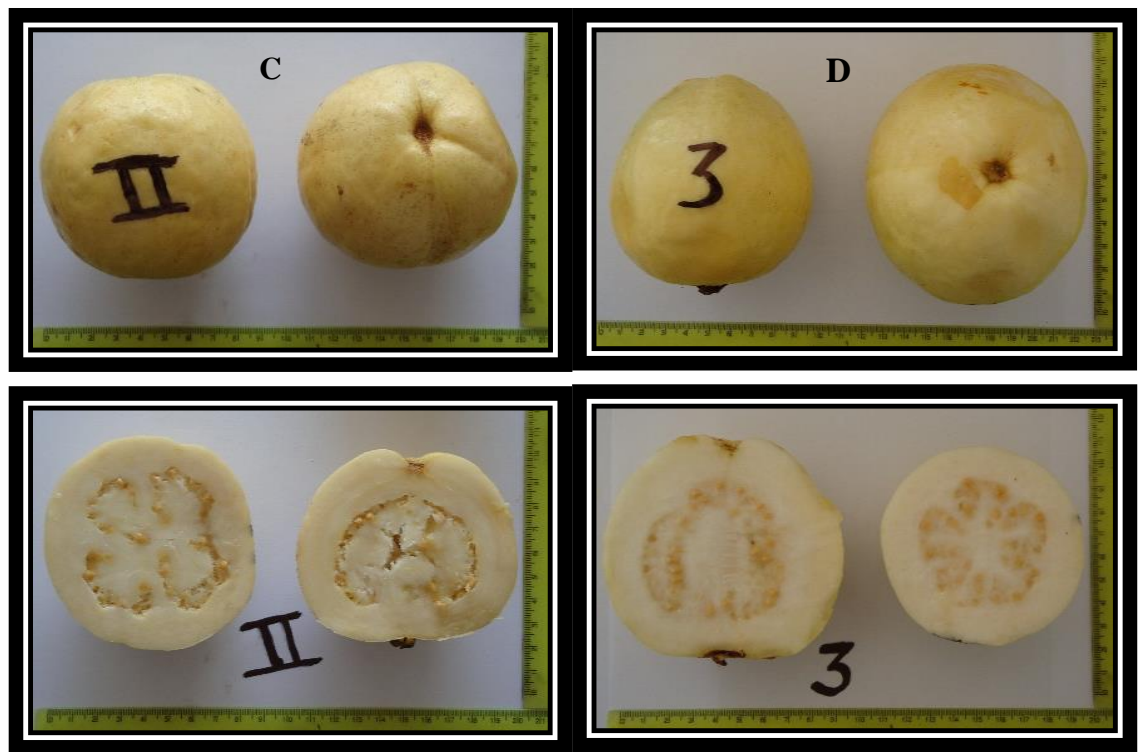

Fig. 2. Guava fruit of some selected strains from orchards C and D

Egypt. J. Hort. Vol. 42, No. 1 (2015) 


\section{References}

A.O.A.C. (1990) Association of Official of Agriculture Chemists, "Official Methods of Analysis", $11^{\text {th }}$ ed. Published by the A.O.A.C. Washington D.C., U.S.A., pp.915, 918, 1048 and 1058.

El-Hamady, M.A., Aida M. Allam, Nasser, M.A. and Hoda A. Galal (2002) Selection and evaluation of some superior seedling guava trees. $2^{\text {th }}$ Inter. Conf. Hort. Sci., 10-12 Sept., Kafr El-Sheikh, Tanta Univ., Egypt.

El-Wakeel, A.T., Mahasen A. El-Badry, Osman, M. and Abu-Nil, S.E. (1971) Possibilities of selection in the seedling progeny of Egyptian guavas. Agric. Res. Review, 49 (5), 139-146.

Gatambia, E.K., Gitonga, J.K., Menza, M.K., Njuguna, J.K., Wanjala, S.B., Karumwa, N. and Wasilwa, L.A. (2009) Situation analysis of guava (Psidium guajava L.) production in eastern province of Kenya. Kenya Agri. Rese. Inst. Thika P.O. Box 220, 01000 and Hqts, P.O. Box 57811-00200 Nairobi, Kenya.

Gomez, K. A. and A. A. Gomez (1984) "Statistical Procedures for Agricultural Research", $2^{\text {th }}$ ed. John Wiley, New York, USA.

Nakasone, H. Y. and R. E. Paull (1998) Guava, In: Tropical Fruits, H.Y. Nakasone and R.E. Paull (Ed.), pp. 149-172, Wallingford, CAB International.

Pommer, C.V. and Katia R.N. Murakami (2009) Chapter 3: Breeding Guava (Psidium guajava L.) In: Breeding Plantation Tree Crops: Tropical Species, Part I: fruit and Nut Crops, S.M. Jain, P.M. Priyadarshan (Ed.), pp: 83-120, (C) Springer Science+Business Media, LLC,

Patel, R.K., Maiti, C.S., Deka, B.C., Deshmukh, N.A. and Roy, D. (2011) Variability studies in guava (Psidium guajava L.) genotypes for growth, yield and quality attributes at mid-hills of Meghalaya. Indian J. Hill Frmg., 24 (1 \& 2), 24-28.

Purseglove, J.W. (1984) Tropical crops dicotyledons. Volume 1 and 2, Longman group limited, ISBN 0-582-40722-2.

Risterucci, A.M., Duval, M.F., Rohde, W. and Billotte, N. (2005) Isolation and characterization of microsatellite loci from Psidium guajava L. Mol. Ecol. Notes, 5, 824-826.

Rokba, A.M., Ezzat, A.H. and El-Wakeel, A.T. (1976) "Bassateen Edfina" a new selected Guava variety. Egypt J. Hort., 3 (2), 209-219. 


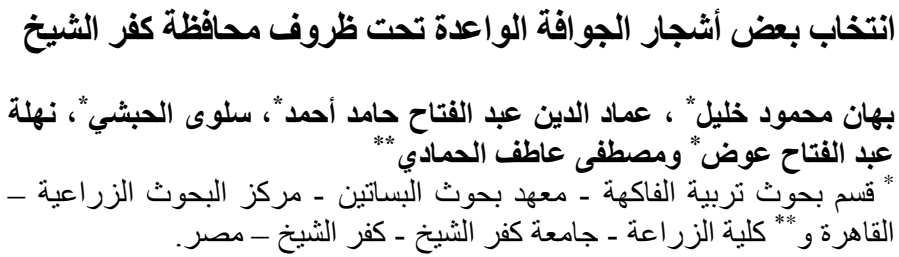

تعتبر الجو افة من الفاكهة شائعة الانتشار في المنطقة الاستو ائية وتحت الاستو ائية،

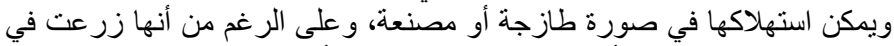

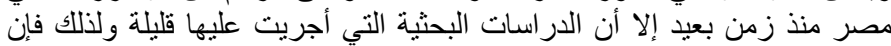
هناك حاجة إلى انتخاب سلالات جيدة لزيادة الإنتاجية لأغر اض الاستهلاك التك الطازج

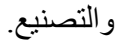

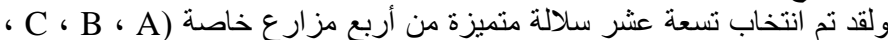

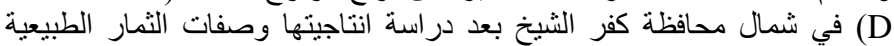

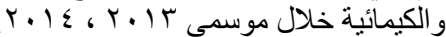

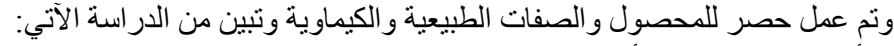

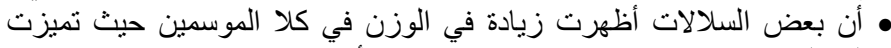

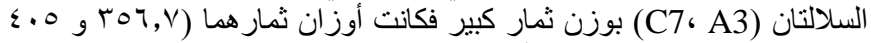

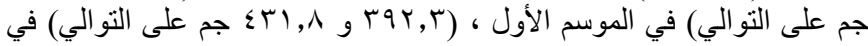

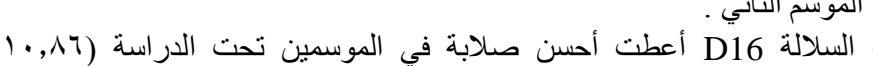

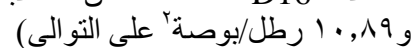

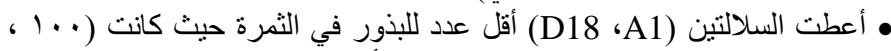

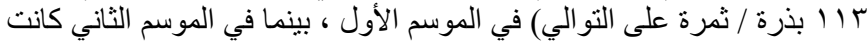

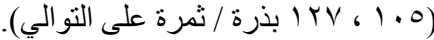

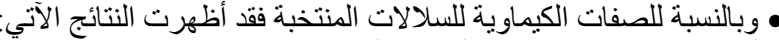

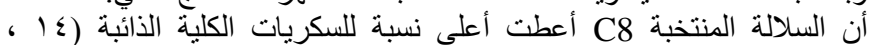

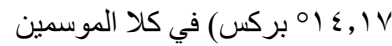

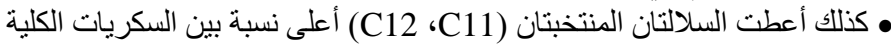

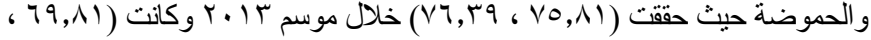

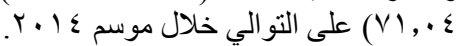

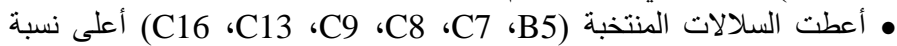
لفيتامين 